Original Research Paper

\title{
Reconceptualization of Glossalgia Issue, Solution Approaches
}

\author{
Alexei Valerievich Yumashev, Irina Mikhailovna Makeeva, \\ Alexander Grigorievich Volkov, Anatolij Sergeevich Utyuzh and Irina Valerievna Nefedova \\ Sechenov First Moscow State Medical University, Moscow, Russia
}

Article history

Received: 30-06-2016

Revised: $24-08-2016$

Accepted: 21-11-2016

Corresponding Author:

Alexei Yumashev

Sechenov First Moscow State

Medical University, Moscow,

Russia

Email: umalex99@gmail.com

\begin{abstract}
The article discusses current therapeutic trends in glossalgia treatment, clinical experimental justification of mesodiencephalon modulation as an effective physiotherapy method with regard to the above abnormality. The principal research method is a clinical experimental one. Major trends in glossalgia treatment have been marked out in the course of scientific search and analysis of theoretical data. Most common physiotherapy methods applied to treat glossopyrosis have been compared. By applying a deductive approach and considering basic etiopathologic mechanisms involved in the pathologic process, we have made a hypothesis of feasibility of the suggested treatment technique. This hypothesis was proved by clinical experiment, while recording and assessing qualitative and quantitative laboratory diagnostic parameters. Modern scientific views of glossalgia, its basic development mechanisms and treatment approaches have been described. Key principles of providing combined medical care to glossalgia patients have been established. Advantages and disadvantages of physiotherapeutic techniques used to treat glossalgia have been discussed. The efficiency of mesodiencephalon modulation as a part of combined treatment of burning tongue syndrome has been justified in theory and proved in practice. Application of mesodiencephalon modulation in glossodynia therapy allows for achieving more relevant clinical outcomes, as compared to other physiotherapeutic methods, while it is distinguished by a high etiopathogenic affinity to the above abnormality. Implementation of this technique is feasible and acceptable for broad clinical use. Due to incorporation of this technique into combined glossalgia treatment, medication load, treatment time, relapse rates can be reduced and quality of patients' life can be improved.
\end{abstract}

Keywords: Glossalgia, Burning Tongue Syndrome, Mesodiencephalon Modulation, Clinical Experiment, Xerostomia, Physiotherapy, Psychoemotional Status, Therapeutic Effect

\section{Introduction}

Glossalgia (burning tongue syndrome, glossodynia, glossopyrosis, or vegetative glossoneurosis) is a polyetiologic disease of the oral cavity that involves prolonged pain and/or paresthesia syndromes of oral localization against no visually detectable pathologic changes in the glossal area. This abnormality is a kind of stomalgia (mouth burning syndrome) and a complex medical issue, which specialized experts often associate with ANS dysfunction, not uncommonly considering glossalgia to be a sensory glossoneurosis. Treatment of burning tongue syndrome patients is one of the most complex and relevant issues of contemporary dentistry and neurology (Yavorskaia, 2007).

As opposed to earlier therapeutic approaches that mostly implied local therapy of this disorder, modern scientific views of glossalgia, major development mechanisms and treatment approaches are marked out by comprehensive understanding of this issue. Despite a broad spectrum of treatment methods and prevailing consensual and successive principle of care provision, therapeutic outcomes with regard to glossalgia are not currently marked by any significant achievements, i.e., full remission rates for treatment of this abnormality is rather low, while recurrences are not rare. We believe 
this to be due to insufficient pathogenic affinity of the applied treatment methods to key links of glossalgia pathogenesis. Moreover, modern clinical practice lacks a unified standardized approach to therapy of this disorder. Therapeutic schedules applied vary greatly, while often involving a great variety of treatment methods and pharmaceutical groups of unproved efficiency towards glossalgia. A polypragmasy phenomenon is not so rare to this effect.

Etiopathogenesis of this disorder is still poorly explored, while this issue is the key to understanding the nature of glossodynia and searching for effective therapeutics for treatment of such disorder. A stress mechanism is one of key mechanisms of glossalgia development. As a result of earlier research, we discovered a high affinity of mechanisms of physiotherapeutic mesodiencephalon modulation technique to systems of pro- and anti-stress regulatory complexes (Yumashev et al., 2016a; Samusenkov et al., 2016). This fact served as the ground for origin of a scientific hypothesis of this method being efficient for treating burning tongue syndrome.

Thus, tasks of this research were as follows:

- Compiling a brief review of major scientific views of ways of glossalgia development

- Performing a comparative analysis of physiotherapeutic treatment methods that are relevant for current clinical practice against major pathogenic aspects of the disorder

- Providing a clinical experimental justification of mesodiencephalon modulation as an effective cutting-edge physiotherapeutic tool in combined glossalgia therapy

\section{Approach}

\section{Theoretical Research}

The first stage of our research involved search and fact-finding and scientific analysis of specialized literature dedicated to the issue of nature of this abnormality and treatment methods. Conformity to the relevant topic and relevance were also accounted for, while selecting materials for review within this research. As a result, we chose and described three major theories of glossalgia development. This allowed us to outline, by deduction and analysis, basic etiopathogenic aspects of this disorder that were used as the basis for our second stage-comparative analysis of most common physiotherapeutic techniques in treatment of this disorder, while specifying therapeutic effects, pros and cons of each approach.

The final stage of this research was a logic continuation of the first two stages and was dedicated to the ultimate goal of this research, i.e., making a hypothesis about the efficiency of mesodiencephalon modulation in glossalgia treatment with experimental verification to follow. The theoretical part of the third stage was fulfilled on the basis of inquiries obtained at earlier stages of this research and was in complete conformance with etiologic principles stated in the first part of this article.

\section{Practical Research}

Experimental stage of this research was done at a specialized medical center that applies Mesodiencephalon Modulation (MDM) technique for system therapy of a variety of abnormalities-MDM centrum clinic in Moscow. This article describes what we believe to be most striking clinical cases of treatment glossalgia patients using this method. All patients, whose data were used in this research, provided their written informative consent to participate in the research.

Sessions were administered using MDM-2000/1 device that delivered pulse currents to subcortex and brain stem (mesodiencephalon). This device is equipped with special protection against electric shock of international IBF class. Operation of this device and procedure administration was entrusted to trained medical personnel and Windows OS SW was used for this purpose.

Technically, an MDM procedure was performed by pulse stimulation of mesodiencephalon of a patient by currents of carrier frequency $10,000 \mathrm{~Hz}$ modulated in LF range 20 to $100 \mathrm{~Hz}$. Input current was 0.5 to $4 \mathrm{~mA}$ and duration of each procedure was $30 \mathrm{~min}$. Treatment sessions were administered daily for 10 days, while 2 procedures with at least $6 \mathrm{~h}$ gap were done for the first three days and patients further received 1 procedure per day. Complete treatment course comprised 13 procedures.

The device was turned on immediately before the session by clicking MDM-2000/1 application icon and then application interface was activated by a doubleclick and patient's input and state of each channel were displayed. The patient sat in a chair and two nickel-plated copper head banded electrodes were placed onto patient's head, using a frontal-occipital technique. The anode was placed in the center of the forehead and cathode was placed in the center of the back of neck, while a single-use 16-layer flannel pad that was thoroughly wet with water was placed between the skin and electrode plate. Special selected therapeutic pulses of the current with alternating specifications through the session according to the given program were delivered via electrodes.

Current intensity was set up smoothly, using a PC keyboard. The desired current was set up by tracking patient's feeling of creeping, slight burning or vibration, separate pulses and minor pressure. The device measured electrode resistance automatically in operation and notified the operator by a flashing message, appearing on the display. Functions Run, 
Cancel, Pause, Current Set-Up were used to interrupt the session and continue with the session, after the required adjustment have been done. Time from procedure start to end was tracked by a timer. The device turned off automatically upon session completion.

Minimum physical and technical parameters to ensure positive therapeutic effect were the following: Current frequency $10,000 \mathrm{~Hz}$, including modulation from $20 \mathrm{~Hz}$, current intensity-over $1 \mathrm{~mA}$. A set of clinical physiological investigations to discover a clear harmonizing effect on central neurohumoral regulatory mechanisms were used to establish the above values. If the inputs increased (frequency-over $10,000 \mathrm{~Hz}$, modulation-over $100 \mathrm{~Hz}$, current intensityover $2 \mathrm{~mA}$ ) patients felt a discomfort in the area of electrode placement.

Inputs of original medical records served as a source for the analysis and selection of comparison parameters and were obtained as a result of a standard clinical investigation that involved a preliminary interview, history taking and physical examination. Moreover, the patients were offered to take a questionnaire survey, using psychological questionnaires and certain additional methods of investigation.

To evaluate the efficiency of the suggested physiotherapeutic technique, the following objective indicators were selected. Autonomous balance normalization was judged by findings of heart rate variability evaluation and cardiointervalography according to Bayevskii. The effect on patients' immune status was evaluated by absolute values of $\mathrm{T}$ lymphocytes, T-helpers/inductors and $\operatorname{IgM}$ titers and functional activity of lymphocytes in a Blast Transformation Reaction (BTR), using a Phytohemagglutinin (PHA). Blood oxygenation rate was determined according to total blood hemoglobin, while microcirculatory gas exchange was tested by pulse oxymetry. The dynamics of psychoemotional state of the patients was investigated by a questionnaire survey, using an Abridged Multi-profile Personality Questionnaire (AMPQ) and Well-being, Activity and Mood questionnaire (WAM). The above additional examination methods were done twice: At initial visit of the medical center complaining of glossalgia and againupon completion of the full MDM course. For conveniences, data of instrumental and laboratory examination methods for each case have been presented in separate tables.

Final conclusion about the efficiency of the suggested technique was provided on the basis of comparing the expected MDM effects and data obtained in special methods of examination and in accordance with outcomes of complex clinical evaluation of patient's condition.

\section{Results}

\section{Etiopathologic Aspects of Glossalgia}

We have performed literature review and distinguished between three major etiologic theories that explain glossalgia development, i.e., multi-factorial, psychogenic and idiopathic.

Followers of the polyetiologic origin of the disorder provide a number of factors of local and systemic value to predispose to and cause unpleasant sensations in oral mucosa directly. The following are mentioned among local conditions to predispose to glossalgia development: Physical and chemical irritants, various allergens, bacterial, viral agents and fungi, specifics of protein composition of saliva, poorly adapted orthodontic and orthopedic structures, impaired musticatory muscle tone, associated with bad habits and certain kinds of tick, local impaired innervations (Kazarina, 2000; Kazarina et al., 2008). Researchers pay special attention to xerostomia with regard to the mentioned issue. The following groups of factors may be referred to systemic reasons associated with the tongue burning syndrome: Alimentary deficient (hypo- and avitaminoses, microand macro-element deficiencies); endocrine (diabetes mellitus, thyroid disorder, menopause and other hormone-deficient conditions); hyposalivation (against any autoimmune disorder, stress and as a result of iatrogeny); medication-induced (due to adverse effects of ACE inhibitors, antipsychotics, antihistamines, chemotherapeutical, antiretroviral drugs and other groups of drugs); gastrointestinal (associated with digestive disorders, e.g., gastroesophageal reflux disease); neurological (neuropathies, neuralgias); immunological (associated with autoimmune disorders and immunodeficiencies) (Kazarina et al., 2008).

The importance of psychogenic factors in the onset of glossalgia syndrome is beyond doubt. According to a variety of research, unpleasant sensations of oral cavity often appear in patients against an apparent stress, anxious, depressive, asthenoneurotic and hypohondriac states, sleep disorders. At the same time, a positive dynamics is not so rarely correlated with the receipt of a specialized treatment aiming at psychoemotional status stabilization, while glossalgia symptoms exacerbation is observed against co-morbid psychopathology aggravation (Bazyan, 2001).

Psychoemotional disorders are believed to play an important role in modulating appreciation of pain and to amplify or attenuate neural transmission from peripheral pain receptors, while modifying individual pain sensitivity and reducing the threshold, leading to a normal irritant to be perceived as a painful one. A concept of psychogenic glossalgia can be found in a number of publications, which implies a direct connection between the onset of glossalgia symptoms 
and any past psychologically important stress. Moreover, some publications describe a phenomenon of somatization of psychic symptoms, with its mechanisms to be fulfilled through neuropathic modifications in the peripheral and central nervous system, as believed by the authors (Stratakis and Chrousos, 1995).

Idiopathic glossalgias take up the smallest share in total set of scientific research dedicated to this issue. They are usually mentioned in cases, when no association of the above groups of etiologic factors can be provided and glossalgia symptoms cannot be explained by organic changes of oral mucosa and other tongue tissues.

To generalize our findings at the first stage of our research, we come to the conclusion that the following etiopathogenic aspects dominate in modern research of this disorder: Functional nature, existing neurohumoral and metabolic disbalance and syndrome of psychoemotional disturbances. Practical relevance of findings of our scientific analysis of specialized literature is outlining a set of relevant therapeutic methods that are marked out by the highest tolerance and efficiency in glossalgia treatment.

\section{Findings of Comparative Analysis of Physiotherapeutic Methods in Glossalgia Treatment}

According to the majority of authors, glossalgia therapy requires a combined application of therapeutic measures in the form of combination of Drug Therapy (Moura et al., 2008), physiotherapy, psychotherapy, reflexotherapy, hyperbaric oxygenation, hirudotherapy and other methods (Bardenshtein et al., 1998; Borisova, 1995; Puzin et al., 1998). A high therapeutic efficiency of transcutaneous electrical nerve stimulation is mentioned in a number of works (Kazarina, 2000; Maina et al., 2002). As long as functional state of the central and peripheral nervous system and ANS in particular, is given the central place in the disease onset, special attention should be paid to such therapeutic measures that aim at stabilizing and normalizing working activity of the involved central nervous system sections of local, segmental and central level. Physiotherapy presents us with great opportunities for this purpose. An advantage of such methods is the universal nature of their therapeutic effects, no apparent adverse effect and good compatibility with other kinds of treatment. At the moment, electric sleep, Cranial Electrotherapy Stimulation (CES) and Laser Therapy (LT) are used among other physiotherapeutic methods as a part of combined glossalgia therapy.

Electroson-4T, EGASS and ES-10-5 devices are used for electric sleep therapy. Therapeutic action is provided in a lying position on a bed and requires a special room design: Darkening and noise insulation. Electrodes are placed onto patient's head, using an orbital-retromastoid technique and direct pulse right-angled currents at a frequency of 5-160 pulse/sec and duration of 0.2-0.5 msec are delivered. Current intensity increases gradually (based on patient's sensation-from slight prickling to painless vibration) and must not generally exceed $8 \mathrm{~mA}$. 20-40 min long procedures are administered daily or every other day. The expected therapeutic effect is tone normalization of various nervous system sections, systemic blood pressure stabilization, superficial tongue mucosa sensitivity restoration and activity harmonization of coagulant and anticoagulant systems, natural sleep deepening, analgesia development and increase in psychoemotional adaptation (Bardenshtein et al., 1998). Medical contraindications (eye disorders, abnormal skin alterations in the areas where electrodes are placed, stage II B and III circulatory disorders, fevers and psychotic conditions), no stable effect and low method specificity (limited by its effect on the ANS and peripheral vascular net) are mentioned among shortcomings of this technique. Electric sleep does not imply any action upon neurohormonal structures that are involved in the pathologic process. Required prolonged course of treatment is mentioned separately.

A TES technique involves action of direct electric current and right-angled pulses at a frequency of $77.5 \mathrm{~Hz}$ and duration of $3.5 \mathrm{msec}$ with a direct and average pulse current correlation of 2 to 5 to 1 (Lebedev, 1993). The following devices are used for physiotherapeutic glossalgia treatment by applying this method: TRANSAIR-2, TRANSAIR- $4 \mathrm{z}$ and ETRANS-1 of standard performance. This procedure is administered in a sitting or lying position. A pair of electrodes is placed above superciliary arches and another pair of electrodes is placed on mastoid processes. Average treatment course duration for this disorder is ten-twenty 40-minute long sessions administered daily (Vasenev et al., 1993). Disadvantages of this method are due to its technical specifics and include impossibility to place electrodes on damaged skin areas. Limited application of this technique is also determined by basic contraindications, such as an electric cardiostimulator installed, thyrotoxicosis, decompensated cardiovascular disorders, hydrocephaly, brain tumors and neuroinfections. Moreover, a positive effect of the TES does not express itself equally in treatment of glossalgias of different genesis. This fact is indicative of limited therapeutic action within its effect on endorphin structures, while the polyetiologic nature of this disorder requires a universal therapeutic approach. One should also mention that judgment of TES efficiency is mainly subjective, while depending greatly on the level of individual pain sensitivity of a certain patient.

Laser therapy of glossalgia is administered, using LG-75 device, a helium neon laser operating at a wavelength of $632.8 \mathrm{~nm}$. This technique involves action on biological active points (by a focused beam) and mucosa areas of modified sensitivity (by a defocused laser). Duration of therapeutic action on any working area is $30 \mathrm{sec}$. Laser physiotherapy course is $10-14$ sessions 
with first five sessions administered daily and the rest of sessions administered every other day. The stated therapeutic effects imply biostimulation and analgesia (Chizhikova et al., 2015). Medical contraindications (acute and de-compensated cardiovascular disorders, essential cerebrovascular disorders, cancers, decompensated diabetes mellitus, blood disorders and hypotonia), prevailing local nature of the effect provided with no account for systemic pathophysiologic mechanisms that are involved in pathologic circle development in glossalgia can be mentioned among shortcomings of the laser therapy. Technical parameters of this method are standard and do not depend on patient specifics.

\section{Mesodiencephalon Modulation and Tongue Burning Syndrome}

Treatment involving mesodiencephalon modulation is a cutting-edge approach to physiotherapeutic glossalgia treatment. MDM therapy ensures full eradication of disease symptoms in a shorter time, while affecting favorably various etiopathogenic aspects of this disorder. A spectrum of therapeutic action of the suggested method covers the following: Correction of patient's psychoemotional status, normalization of metabolism and morphofunctional tissue status at the local level, analgesia and balance recovery of neurohumoral regulatory systems of the body (Yumashev et al., 2016b).

Through the MDM therapy, weak electrical signals of tailored specifications with view to patient's gender and age, clinical progression and co-morbid abnormality affect brain structures, while activating selectively various parts of the neuroendocrine system, mobilizing immune forces and facilitating optimum use of internal body's reserves, thus balancing out pro- and anti-stress systems (Karev, 2005).

Functioning normalization of the neurohumoral regulatory complex is due to release into the blood stream of certain neuropeptides-stabilizing person's emotional and psychic state. This release helps to eradicate anxiodepressive, hypohondriac and asthenoneurotic phenomena that are typical of glossalgia patients, while limiting or excluding any medication use. Moreover, mesodiencephalon modulation has an apparent analgesic effect, ensuring eradication of abnormal sensitivity symptoms.

Electric stimulation of mesodiencephalic structures primarily ensures normalized activity of the sympathoadrenal complex, i.e., a stress system that provides neurohumoral regulation of vital processes and realizes an adaptive response to any adverse factor, which the disorder actually is. At the same time, MDM has an activating effect on central opioid and serotonergic systems responsible for an antinociceptive effect and psychoemotional regulation. Involvement of GABA-ergic structures and benzodiazepine receptors, being antagonists of catecholamines and having a general sedative, anxyolitic and hypnotic effect, also plays an important role in therapeutic effect development. Current stimulation of mesocortex and limbic structures provides for sanogenic action on emotions and behavior that are prone to adverse changes in glossalgia patients, while facilitating reduced anxious, depressive, neurotic and hypohondriac expressions in such patients (Yumashev et al., 2016b).

Activation of anti-stress mechanisms that are induced by mesodiencephalon modulation helps with stress hypertension (which is not unimportant with view to the concurrent abnormality) and boosts immunocompetence due to optimization of patient's body defenses and rational realization of immune responses. At the local level, the therapeutic effect is based on involvement of the peripheral opioid receptor system mediating analgesia, kallikrein-kinin system having an anti-inflammatory effect and facilitating microcirculation and antioxidant factors, preventing cell alteration (Karev, 2005).

At the systemic level, the therapeutic effect of MDM therapy is normalization of psychosomatic and neurohumoral interactions of glossalgia patient's body, while it is expressed locally in pain and paresthesia management and improvement of tissues' functional status and trophism to be a guarantee of rehabilitating patient's quality of life up to full-rate recovery and local symptoms regression, psychoemotional stabilization and ANS tone normalization (Yumashev et al., 2016b; Utyuzh et al., 2016).

Therefore, the expected MDM therapy effects in glossalgia treatment are polymodal and include the following: Pain and paresthesia sensation management, normalization of sensitivity and tissues' functional state, microcirculation and oxygenation improvement, overall anti-stress action due to attenuated sympathicotonic ANS effect and harmonized activity of immune and neurohumoral regulatory systems and sedation. Overall clinical sanogenic influence of the technique is determined by normalized psychoemotional status, improved overall non-specific body resistance, recovered natural biorhythm and sleep, reduced medication load and shortened treatment time against stable improvement.

\section{Discussion}

Description of Clinical Cases

Case 1

Gender

Female, age 46 years old.

\section{Diagnosis}

Psychogenic glossalgia of paresthetic type. 


\section{Co-Morbidity}

Early surgical climax, hormonal replacement therapy.

\section{Admission Complaints}

Feeling of prickling, presence of a thread or hair that is spread onto external $2 / 3$ of the tongue, irritability, easy crying, migraine-like headaches, hot flashes, insomnia.

According to patient's history, the above symptoms have been gradually developing for 3 years and the patient was earlier examined many times to this effect and diagnosis of psychogenic glossalgia was made.

A course of treatment was administered: Pulse current application to subcortex and brain stem, using a frontal-occipital technique, following assessment of clinical setting.

\section{Current Specification}

Pulse mode at a carrier frequency of $10,000 \mathrm{~Hz}$, modulation in a LF range of 20 to $100 \mathrm{~Hz}$, current intensity of $2.5 \mathrm{~mA}$. Good tolerance of the MDM therapy was observed; no adverse reactions through the course were noted.

\section{Clinical Outcome}

Paresthetic and asthenoneurotic syndrome regression, sleep normalization, reduced manifestation of hot flashes, reduced frequency of headache episodes were observed, the patient herself finds improved overall wellbeing and psychoemotional state.

Along with the subjectively determined improvement, a positive dynamics of patient's clinical state was also observed according to special examination methods (Table 1).

Heart rate variability assessment was used to establish autonomous balance normalization, i.e., the initial ANS state was characterized by prevailing sympathetic activity, while a sympathetic lytic effect was determined after the MDM course up to a balanced autonomous regulation established. Post-treatment Cardiointervalography (CIG) analysis according to Bayevskii showed a decrease in mode amplitude and autonomous balance indexes, as well as regulatory system stress. A vagal shift argued for reduced regulatory body efforts, which may be associated with an increase in functional consistency and expansion of adaptive reserves of patient's body.

Table 1. Physical examination data for clinical case 1

\begin{tabular}{|c|c|c|}
\hline & \multicolumn{2}{|l|}{ Autonomous balance } \\
\hline & Before treatment & After treatment \\
\hline \multirow[t]{8}{*}{ Heart rate variability } & $\mathrm{TF}=2,675 \mathrm{msec}^{*} 2$ & \\
\hline & $\mathrm{LF}=1,342 \mathrm{msec}^{*} 2$ & \\
\hline & $\mathrm{HF}=386 \mathrm{msec}^{*} 2$ & \\
\hline & $\mathrm{VLF}=947 \mathrm{msec}^{*} 2$ & \\
\hline & $\mathrm{LF} / \mathrm{HF}=3,48$ & \\
\hline & $\% \mathrm{LF}=50.2$ & $\% \mathrm{LF}=32.2$ \\
\hline & $\% \mathrm{HF}=14.4$ & $\% \mathrm{HF}=52.9$ \\
\hline & $\% \mathrm{VLF}=35.4$ & $\% \mathrm{VLF}=15$ \\
\hline \multirow[t]{3}{*}{ Cardiointervalography acc. to Bayevskii } & $\mathrm{AMo}=54 \%$ & $\mathrm{AMo}=28.9 \%$ \\
\hline & $\mathrm{ABI}=138$ rel. un. & $\mathrm{ABI}=12.3$ rel. un. \\
\hline & $\mathrm{SI}=123.1$ rel. un. & $\mathrm{SI}=6.62$ rel. un. \\
\hline \multicolumn{3}{|l|}{ Immune status } \\
\hline T-lymphocytes (abs.) & $619.9 * 10^{6} / \mathrm{L}$ & $1,242.6^{*} 10^{6} / \mathrm{L}$ \\
\hline T-sub-population (abs.) & $112.3^{*} 10^{6} / \mathrm{L}$ & $177.0^{*} 10^{6} / \mathrm{L}$ \\
\hline T-helpers/inductors & $13.9 \%$ & $16.41 \%$ \\
\hline T-helpers (abs.) & $150 * 10^{6} / \mathrm{L}$ & $243 * 10^{6} / \mathrm{L}$ \\
\hline BTR, using PHA & $17,855.3 \mathrm{pulse} / \mathrm{min}$ & $43,892.4 \mathrm{pulse} / \mathrm{min}$ \\
\hline $\operatorname{Ig} \mathrm{M}$ & $1.7 \mathrm{~g} \mathrm{~L}^{-1}$ & $1.6 \mathrm{~g} \mathrm{~L}^{-1}$ \\
\hline \multicolumn{3}{|l|}{ Blood oxygenation } \\
\hline $\mathrm{Hb}$ & $118 \mathrm{~g} \mathrm{~L}^{-1}$ & $134 \mathrm{~g} \mathrm{~L}^{-1}$ \\
\hline \multicolumn{3}{|l|}{ Microcirculation } \\
\hline $\mathrm{Sp}\left(\mathrm{O}_{2}\right)$ & $94 \%$ & $98 \%$ \\
\hline
\end{tabular}

TF-Total spectrum power; LF-Low-Frequency component; HF-High-Frequency component; VLF-Very Low-Frequency component; LF/HF-vagosympathetic cooperation index; \%LF-relative Low-Frequency component value; \%HF-relative high-frequency component value; \%VLF-relative very low-frequency component value; BTR, using PHA-blast transformation reaction, using a phytohemagglutinin; (abs.)-absolute content; Ig M -macroglobulin titer; AMo-mode amplitude ; ABI-Autonomous Balance Index; SI-Stress Index; Hb-total hemoglobin; $\mathrm{Sp}\left(\mathrm{O}_{2}\right)$-peripheral oxygen saturation 
Immune status examination revealed a post-treatment growth of absolute T-lymphocyte and T-sub-population content, T-helper/inductor content also increased and absolute T-helper value grew. An accelerated positive PHA-based BTR dynamics was observed. Macroglobulin titers decreased after MDM course completion.

An increase in total blood hemoglobin along a growth of peripheral oxygen saturation was observed through the treatment. These data argue strongly for increased blood oxygen capacity and oxygen saturation of peripheral tissues.

Improved patients' psychological state was discovered using an AMPQ, i.e., a decrease in profiles according to the second scale (from 68 down to 46) and fourth scale (from 57 down to 50) was observed, which expressed itself in increased psychological, adaptivity, decreased proneness to conflicts, impatience and aggression. According to WAM test, truly improved well-being, activity and mood was determined.

\section{Case 2}

\section{Gender}

Male, age 36 years old.

\section{Diagnosis}

Glossalgia of mixed paresthetic and allergic type.

\section{Admission Complaints}

Dragging pain, feeling of numbness and prickling across the whole tongue surface that intensify in stress, unstable mood, increased fatigue, irritancy, excessive palm sweating, anxious feeling, palpitation when feeling worried.

According to the history, the above symptoms have been present for 6 years and that is why the patient sought medical care, was thoroughly examined and no organic abnormality was discovered, glossalgia diagnosis was made. The patient underwent out-patient treatment three times and the positive effect was not stable.

With view to the above, a MDM course was administered, i.e., pulse current application to subcortex and brain stem (mesodiencephalon), using a frontaloccipital technique.

\section{Current Specification}

pulse mode at a carrier frequency of $10,000 \mathrm{~Hz}$, modulation in a LF range of 20 to $100 \mathrm{~Hz}$, current intensity of $1.8 \mathrm{~mA}$. Tolerance of electric current action was good, no adverse reactions through the treatment were observed.

\section{Clinical Outcome}

Significant pain syndrome regress, eradication of paresthetic sensations, improved overall well-being of the patient, mood flattening, increased overall tone, decreased manifestations of irritancy and autonomous lability.

Along with the subjectively determined improvement, a positive dynamics of patient's clinical state was also determined according to special examination methods (Table 2).

Table 2. Physical examination data for clinical case 2

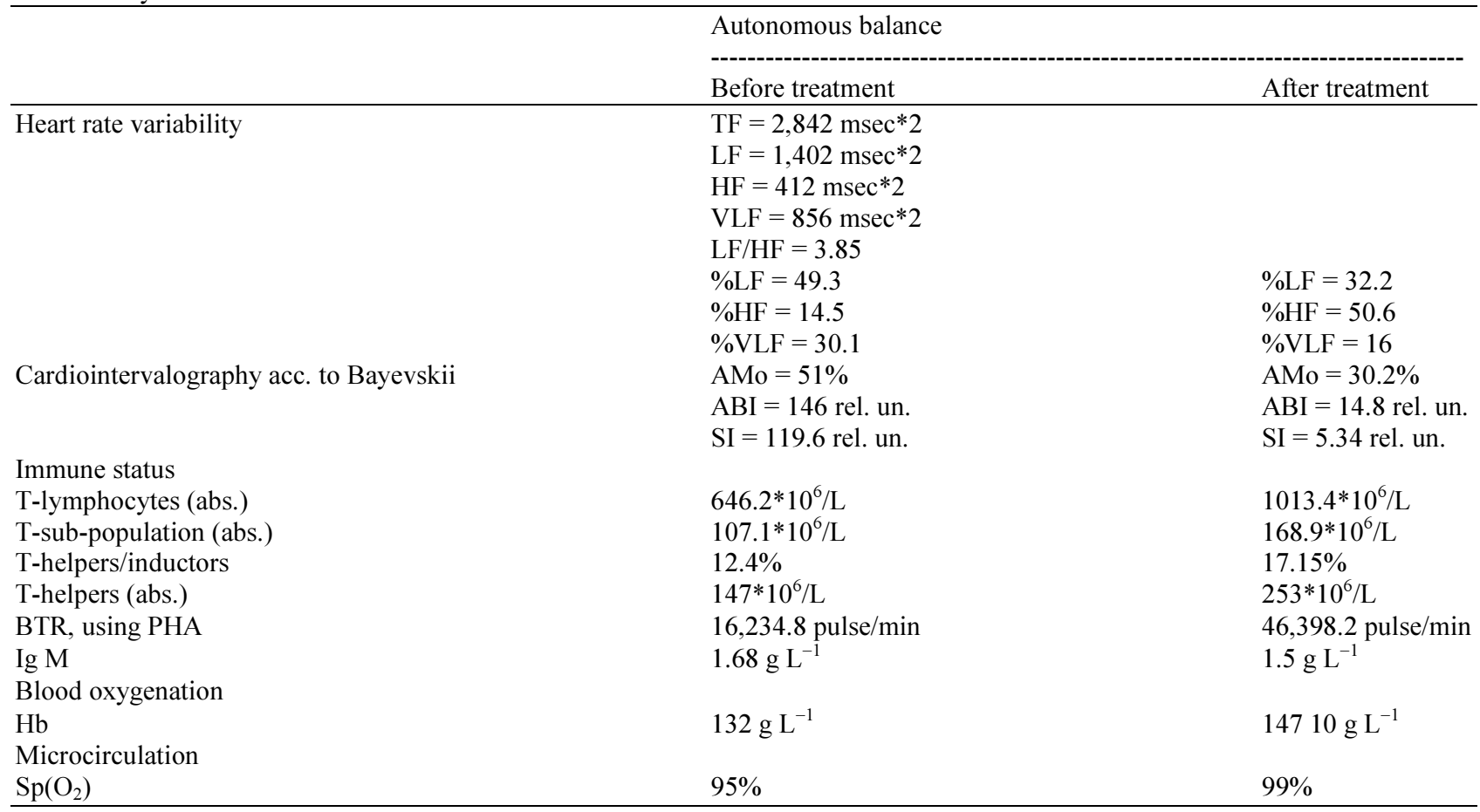


Autonomous balance was assessed by CIG according to R.M. Bayevskii. The initial ANS was characterized by prevailing parasympathetic activity. Reduced intensity of the parasympathetic effect up to establishment of a balanced autonomous regulation type was observed after the correction course.

Immune status examination revealed increased absolute T-lymphocyte and T-sub-population content after the MDM course, a growth of T-helper/inductor content and absolute T-helper value. A clear PHA-based BTR dynamics was also registered. Macroglobulin titers decreased after treatment completion.

According to general blood count, total hemoglobin increased, as compared to the initial value. Peripheral oxygen saturation increased, according to pulse oxymetry indicating a positive effect on oxygenation and microcirculation.

Improved patient's psychoemotional state, based on AMPQ data, expressed itself in decreased indicators according to anxiety triad scales $(1,2,7)$. WAM test data also genuinely attested to improved well-being, activity and mood, i.e., profiles decreased according to the first scale from 56 down to 47 , according to the second scale from 59 to 51 and according to the seventh scale from 58 to 50 , which was reflected in improved psychological adaptation, decreased proneness to conflict, impatience and aggression.

\section{Case 3}

\section{Gender}

Male, age 30 years old.

\section{Diagnosis}

Glossalgia.

\section{Admission Complaints}

Feeling of burning across the external tongue surface, pain that intensifies during meals.

According to the history, the above symptoms have been the trouble for 3 years, following an orthodontic treatment for a year, the patient was examined may times by a neurologist, dentist, psychotherapists (a course of antidepressants was prescribed, having minimum effect), glossalgia diagnosis was made.

With view to the above, a course of treatment was administered, i.e., application of pulse currents, using MDM-2000/1 device, to subcortex and brain stem (mesodiencephalon), using a frontal-occipital technique.

\section{Current Specification}

Pulse mode at a carrier frequency of $10,000 \mathrm{~Hz}$, modulation in a LF range of 20 to $100 \mathrm{~Hz}$, current intensity of $2 \mathrm{~mA}$. Tolerance of procedures was good, no adverse reactions were observed.

\section{Clinical Outcome}

After the treatment, pain syndrome and burning regression was observed; patient's well-being improved greatly, psychoemotional and autonomous disorders decreased.

A positive dynamics of patient's conditions was also proved by special tests, along with subjective feelings (Table 3).

Table 3. Physical examination data for clinical case 3

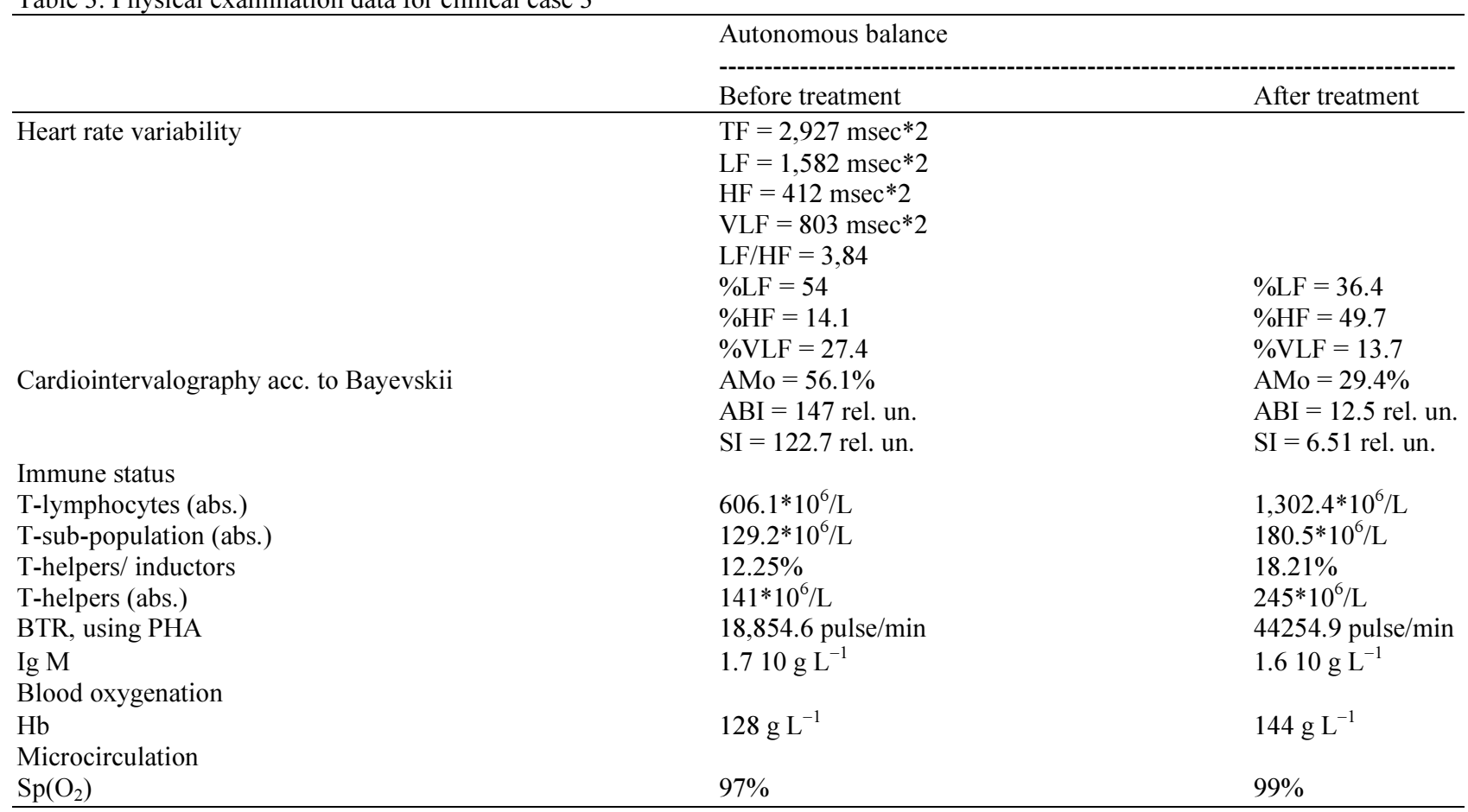


Heart rate variability test data argued for autonomous balance normalization, i.e., initial state of the ANS was characterized by prevailing sympathetic activity, while a decrease in sympathetic effects was observed after the correction course with a balanced autonomous regulation established.

According to CIG analysis according to Bayevskii, a vagal shift was detected after the treatment, which was indicative of reduced regulatory body efforts and increased performance with an ability to expand adaptive reserves of patient's body.

According to immune status examination, initially reduced absolute T-lymphocyte level grew to normal, absolute indicators of T-lymphocyte sub-population and number of T-helpers/inductors also increased, absolute T-helper value grew significantly. An accelerated PHAbased BTR dynamics was observed. Macroglobulin titer slightly decreased through the treatment.

According to total blood count, increased blood oxygenation was observed based on an incremental growth of total hemoglobin. Improved microcirculation and peripheral gas exchange was determined by pulse oxymetry, which is indicative of increased peripheral oxygen saturation after the MDM course.

A positive dynamics of patients' psychological state was discovered according to AMPQ data, i.e., profiles decreased according to the second scale from 67 down to 47 and according to the fourth scale from 58 down to 52 , which expressed itself in decreased proneness to conflict, impatience and aggression. According to WAM test, truly improved well-being, activity and mood was determined.

\section{Conclusion}

We have confirmed the suggested hypothesis of MDM efficiency in combined glossalgia treatment and have come to the following scientific conclusions as a part of our research:

Modern etiopathogenic views of glossalgia, as opposed to earlier concepts, are marked by a broad clinical view and come down to three etiologic theories, i.e., multi-factorial, psychogenic and idiopathic theories that prevail in specialized literature.

Optimized metabolism, improved innervation and blood flow in tissues, disease symptoms regression are expected therapeutic effects in combined glossalgia treatment by such physiotherapeutic methods as electric sleep, electric neurostimulation and laser therapy. However, lack of clear conformity of therapeutic effects of such procedures and etiopathogenesis of this abnormality, very specific action of such procedures along with certain medical contraindications make it impossible to approve such kinds of treatment for broad clinical use.

An advantage of the MDM therapy, as compared with other physiotherapeutic methods of glossalgia treatment is clearly traceable versatile nature of therapeutic action covering different etiopathogenic mechanisms involved in development of this abnormality.

According to clinical outcomes, MDM therapy is an effective method of treatment of this abnormality, which is confirmed by subjective condition improvement and data of objective instrumental and laboratory methods of examination.

Mesodiencephalon modulation can be recommended to be incorporated into the combined glossalgia therapy system as an effective and safe treatment method. Speaking of our findings again, it should be mentioned that MDM therapy is virtually a new concept of sanogenesis involving all three major therapeutic principles, i.e., etiological, pathogenic and symptomatic, thus being of special interest for the purpose of curing glossalgia patients completely. Affordability, no-medication nature and good compatibility with other types of treatment and ability to amplify the effect of medications are additional advantages of this method. It allows to avoid polypragmasy, while reducing toxic load on the glossalgia patient's body significantly, with such patients (with view to the complex nature of this disease) often subjected to massive pharmacotherapy, including anti-bacterial, anti-fungal, anti-inflammatory, pain-relieving, immunomodulating, sedative, psychoactive and other groups of drugs. MDM application facilitates reduction in rates of toxic, allergic, gastrointestinal, cardiovascular, extrapyramidal and other adverse effects in glossalgia patients, which affects favorably their overall condition, psychoemotional status and quality of life at no expense of treatment efficiency. No broad spectrum of contraindications, good tolerance and safety of the procedure and ability to adjust technical inputs as tailored to gender and age and clinical inquiry are also benefits of this technique.

The above arguments argue for the necessity to review current care standards for glossalgia patients to possibly incorporate the suggested technique into the combined treatment.

\section{Acknowledgment}

The authors would like to acknowledge the assistance of Oleg. I. Admakin, Professor, Dean of the Faculty of Dentistry, Head of the Department of Prevention and Community Dentistry, Sechenov First Moscow State Medical University.

\section{Author's Contributions}

The authors have equally contributed to the conducted research and writing of the article.

\section{Ethics}

This article is original and contains unpublished material. The authors confirm that there are no ethical issues involved. 


\section{References}

Bardenshtein, L.M., A.B. Klimov, M.G. Panin and V.V. Mikhailova, 1998. The efficiency of medicamental correction of psychopathologic conditions in patients with facio-maxillary deformations. Neurodentistry Dentistry, 1: 52-56.

Bazyan, A.S., 2001. Interaction between transmitting and modulating systems of the brain and their possible role in creating psycho-physiological and psychopathological conditions. Success Physiol. Sci., 32: 3-22.

Borisova, E.G., 1995. Arranging out-patient care for neurodental patients. Dentistry, 5: 68 .

Chizhikova, T.S., S.V. Dmitrenko, R.D. Yusupov and T.V. Chizhikova, 2015. The efficiency of combined treatment of glossalgia and burning mouth syndrome. Kuban Med. Res. Bull., 1: 128-132.

Karev, V.A., 2005. Mesodiencephalic modulation. Moscow.

Kazarina, L.N., 2000. Glossalgia. In: Diseases of the Oral Mucosa, Nizhnii Novgorod, pp: 227-239.

Kazarina, L.N., L.M. Vdovina and A.I. Volozhin, 2008. Glossalgia: Etiology, Pathogenesis, Clinical Picture and Treatment. Nizhnii Novgorod.

Lebedev, V.P., 1993. A New method of Nonpharmaceutical Treatment. Saint-Petersburg.

Maina, G., A. Vitalucci, S. Gandolfo and F. Bogetto, 2002. Comparative efficacy of SSRIs and amisulpride in burning mouth syndrome: A singleblind study. J. Clin. Psychiatry, 63: 38-43. DOI: $10.4088 / J C P . v 63 n 0108$

Moura, M.D., M.I. Senna, D.F. Madureira, L.M. Fonseca and R.A. Mesquita, 2008. Oral adverse effects due to the use of nevirapine. J. Contemporary Dental Pract., 9: 84-90. PMID: 18176653
Puzin, M.N., G.M. Nesterenko, N.V. Grishina and T.V. Morozova, 1998. New Information about Burning Mouth Syndrome [according to national and foreign publications]. Neurodentistry Dentistry, 1: 47-52.

Samusenkov, V.O., A.S. Utyuzh, A.V. Yumashev, I.V. Nefedova and M.S. Podporin, 2016. Untersuchung der stabilität der zahnimplantate. Europäische Fachhochschule, 5-6: 28-30.

Stratakis, C.A. and G.P. Chrousos, 1995. Neuroendocrinology and pathophysiology of the stress system. Ann. NY Acad. Sci., 771: 1-18. DOI: $10.1111 /$ j.1749-6632.1995.tb44666.x

Utyuzh, A.S., V.O. Samusenkov, A.V. Yumashev, I.V. Nefedova and T.V. Tsareva, 2016. Analysis of osseointegration adequacy and examination of stability of dental implants after sinus lift operation. Austrian J. Tech. Natural Sci., 5-6: 16-19.

Vasenev, Y.E., N.V. Morozova and V.F. Mikhalchenko, 1993. The efficiency of cranial electrotherapy stimulation in combined therapy of chronic pain syndrome of the oral cavity. Department at the National Central Medical Research Library of the RF, D-23679:6.

Yavorskaia, Y.S., 2007. Pain and paresthetica syndromes of the maxillo-facial area. Medkniga, Kiev.

Yumashev, A.V., T.N. Gorobets, A.S. Utyuzh, G.G. Kuzminov and I.V. Nefedova, 2016b. Klinische und pathologisch-genetische begründung der anwendng des mdm-verfahrens in der korrektur von stressstörungen. Europaische Fachhochschule, 4: 8-12.

Yumashev, A.V., T.N. Gorobets, O.I. Admakin, G.G. Kuzminov and I.V. Nefedova, 2016a. Key aspects of adaptation syndrome development and anti-stress effect of mesodiencephalic modulation. Ind. J. Sci. Technol., 9: 1-10. DOI: $10.17485 /$ ijst/2016/v9i19/93911 